\title{
Uma ferramenta móvel para recomendação de visualização de dados abertos municipais
}

\author{
Marcelo Vitório $^{1}$, Raissa Barcellos ${ }^{1}$, Flavia Bernardini ${ }^{1}$, José Viterbo ${ }^{1}$ \\ ${ }^{1}$ Instituto de Computação - \\ Universidade Federal Fluminense (UFF) - \\ Niterói - RJ - Brasil \\ \{rbarcellos, marcelovitorio\}eid.uff.br \\ \{fcbernardini,viterbo\}eic.uff.br
}

\begin{abstract}
Tasks performed by government agencies produce exorbitant amounts of raw data daily. However, the value that these data could provide for society's different sectors can be lost if they are not properly collected and managed. Even with the adoption of data opening policies, institutions still face problems in the efficient use and interpretation of this data due to lack of training, appropriate tools, or cultural factors. In this work, we propose the construction of a mobile application to view data from the Open Data Portal in Recife. The application is based on an open data visualization recommendation framework, which can analyze the data types of the fields in a given data set to make the appropriate visualization type recommendation.
\end{abstract}

Resumo. As tarefas realizadas por órgãos governamentais produzem quantidades exorbitantes de dados brutos diariamente. Entretanto, o valor que estes dados poderiam proporcionar para os diversos setores da sociedade pode se perder caso estes não sejam devidamente coletados e gerenciados. Mesmo com a adoção de políticas de abertura de dados, as instituições ainda enfrentam problemas na utilização eficiente e interpretação destes dados por conta de problemas como falta de capacitação, ferramentas apropriadas ou fatores culturais. Neste trabalho propomos a construção de um aplicativo mobile para visualizar dados provenientes do Portal de dados abertos do município de Recife. $O$ aplicativo é baseado em um framework de recomendação de visualizações de dados abertos, que é capaz de analisar os tipos de dados dos campos de um determinado conjunto de dados a fim de realizar a recomendação do tipo de visualização apropriada.

\section{Introdução}

Governos de todo mundo estão cada vez mais buscando maneiras mais eficazes de gerenciar dados para apoiar suas atividades e atender melhor às demandas da sociedade como um todo [Andrews 2019]. Os sistemas de dados integrados, que coletam informações em tempo real de várias agências, podem ajudar os gerentes a obter percepções adicionais sobre os problemas, porém a falta de capacidade de construir tais sistemas pode obrigar essas entidades a usar sistemas manuais para rastrear processoschave [Farazmand et al. 2018]. Apesar do movimento de dados abertos ganhar força, a 
falta de recursos essenciais e habilidades podem bloquear a utilização efetiva dos dados [Gurstein 2011]. No contexto da gestão pública, a refatoração de processos e o desenvolvimento de novos produtos, se dá através da inovação orientada a dados que mesmo com sua necessidade sendo evidenciada ainda encontra barreiras no âmbito público por conta de diversos fatores [Cronholm et al. 2017], [Brandão and Bruno-Faria 2017]. Os autores em [Kerr do Amaral and Licio 2008], enfatizam que o principal catalisador da inovação na gestão pública é o gestor, pois este possui as ferramentas necessárias para alavancar a mudança dentro da organização. Contudo, alguns gestores ainda não compreendem os benefícios da utilização dos dados e assim a utilização dos dados no setor público permanece muito limitada [Guenduez et al. 2020].

Com o avanço da tecnologia e aumento da utilização de dispositivos mobile pela população em geral a facilidade e a rapidez no consumo de informações alterou completamente a forma como tomamos decisões e vemos o mundo a nossa volta [Paraskevopoulos et al. 2013]. Tais fatores preconizam a concepção de que toda informação deve estar ao nosso alcance a todo momento e de que a realização de quase todas as tarefas pode ser feita com alguns cliques.

Levando em consideração a falta de sistemas para utilização efetiva dos dados nas organizações públicas e a utilidade que uma ferramenta mobile pode trazer ao consumir informações por conta de sua ubiquidade, este trabalho tem como objetivo incentivar a utilização de ferramentas de tomada de decisão, por parte dos gestores públicos, através de visualizações de qualidade, entretanto, antes precisamos compreender melhor os desafios que permeiam a implementação de ferramentas desse cunho no âmbito público, o que é realizado na Seção 2 deste estudo. Já na Seção 3, é descrita a revisão da literatura e ferramentas semelhantes. Na Seção 4, o escopo e a utilização da ferramenta desenvolvida são apresentados e a Seção 5 conclui o estudo detalhando as contribuições da ferramenta, seus aperfeiçoamentos futuros e possíveis evoluções.

\section{Referencial Teórico}

\subsection{Dados governamentais abertos}

O crescimento exponencial da produção de dados e a tendência social de abertura e compartilhamento são forças poderosas que estão mudando a economia e a sociedade [Bernardini et al. 2020]. Governos de todo o mundo tornaram - se participantes ativos dessa evolução, abrindo seus dados para acesso e reutilização por agentes públicos e privados [Jetzek et al. 2014]. De acordo com a European Data Portal ${ }^{1}$ dados abertos podem ser definidos como dados que qualquer pessoa pode acessar, usar e compartilhar. Devendo ser disponibilizados em um formato legível por máquina e licenciados de forma que sua utilização seja totalmente livre.

No entanto, segundo [Zuiderwijk and Janssen 2014], os governos estão mais focados em divulgar dados indiscriminadamente, do que realmente estão envolvidos em busca de meios para melhorar a formulação de políticas de dados abertos e como lidar, de fato, com riscos de todo o processo de abertura de dados, como violação de privacidade direta ou indireta, responsabilidade legal e má interpretação de dados. O incentivo pela disponibilização e o aumento na procura a dados abertos partem da compreensão de que

\footnotetext{
${ }^{1} \mathrm{https}: / / \mathrm{www}$. europeandataportal.eu
} 
a disponibilização de dados é apenas parte de uma iniciativa que visa praticar a transparência das contas públicas e promover a compreensão e participação do cidadão nas decisões governamentais. Conceitos como dados abertos, governo aberto, transparência e participação da população são interligados por meio de uma série de recursos e tecnologias, evidenciando a necessidade de ferramentas para auxiliar às pessoas no acesso, compreensão e manipulação desses dados [Macedo et al. 2020].

\subsection{Gestão pública orientada à dados}

A necessidade de coletar, filtrar e interpretar grandes volumes de informações faz parte da vida moderna - e parte do governo moderno [Farazmand et al. 2018]. Essa necessidade surge a partir das atividades e processos de uma organização pública que acabam gerando de forma passiva uma grande quantidade de dados diariamente, entretanto, o valor desses dados muitas vezes pode ser negligenciado por falta de capacitação, ferramentas apropriadas ou fatores culturais. Gestores que buscam fazer escolhas efetivas para alcançar o objetivo de sua organização podem encontrar o alicerce de uma decisão bem sucedida nestes dados gerados que não foram devidamente coletados e analisados. A era do big data trouxe desafios para liderança pública em diferentes jurisdições [Andrews 2019]. Nessa conjuntura, surge o conceito de Gestão orientada à dados, que é definida por [Farazmand et al. 2018] como o uso estratégico e rotineiro dos dados para melhorar a qualidade do serviço e alcançar os objetivos chave de uma organização.

Para utilizar efetivamente os dados de forma que orientem suas decisões, os líderes precisam de informações consistentes sobre as operações sob sua jurisdição [Farazmand et al. 2018]. Porém, a capacidade de coleta de dados, apesar de demandar bastante eficácia e inteligência, cresce rapidamente enquanto a capacidade de análise desse grande volume de dados aumenta lentamente [Keim et al. 2006]. Para se adaptar a esse novo contexto, o desenvolvimento de processos e ferramentas capazes de extrair e analisar esses dados serão diferenciais para organizações que buscam promover a rápida tomada de decisão e aumentar a produtividade visto que os ambientes externos das organizações afetam o funcionamento interno das mesmas e vice-versa. Por esse motivo, elas precisam ser mais flexíveis e atentos às mudanças externas para responder a elas [Ferreira 2019].

Tendo em vista que a realidade do serviço público é permeada por desafios complexos, a criatividade no desenvolvimento de novos métodos e processos de gerenciamento, que visam aumentar a capacidade de resposta do governo em resolver problemas e atender a necessidades coletivas, faz - se necessária por parte dos agentes públicos (ENAP, 2013). Exatamente por conta desses desafios complexos que permeiam a gestão pública a inovação vem ganhando mais espaço como tema estratégico [Magnusson et al. 2020]. Embora possa ser muitas vezes encarada como um artigo de luxo ou um encargo adicional não desejado, [Magnusson et al. 2020] pontuam que a inovação deve ser tratada como uma atividade central na administração pública, pois ela ajuda a desenvolver melhores maneiras de atender necessidades, resolver problemas, utilizar recursos e tecnologias além de aumentar a capacidade de resposta dos serviços às necessidades locais e de acompanhamento das necessidades e expectativas públicas.

No trabalho [Mergel 2018], o autor ressalta a importância do gestor público, concluindo que eles são um dos catalisadores de ambientes favoráveis à inovação devido 
sua posição hierárquica. Em [Nicholson-Crotty et al. 2017], o autor aponta a importância do incentivo ao comportamento inovador por parte dos gestores públicos e alega a relevância da compreensão da percepção de risco por parte dos agentes públicos, sendo a cultura de aversão ao risco descrita por [Bommert 2010] como um limitador fundamental da liderança em seus aspectos substanciais necessários para implementar, gerar e difundir ideias.

\subsection{Plataformas para gestão de dados abertos}

No momento atual, existe uma grande variedade de material científico sobre dados abertos e plataformas de dados abertos de uma perspectiva social, econômica e legislativa. Também dispomos de uma gama de plataformas que apoiam a construção de portais de dados abertos, como as plataformas CKAN, OGDI DataLab, LIBRE, Socrata Open DataPortal e ODS [Machado et al. 2018]. Neste trabalho, utilizamos a API do ckan no desenvolvimento da ferramenta proposta.

\subsubsection{CKAN}

$\mathrm{O} \mathrm{CKAN}^{2}$ é o sistema de gerenciamento de dados abertos, sendo o mais utilizado a nível mundial por usuários de diferentes domínios, mas principalmente pelo setor público, contando com cerca de 199 instâncias. O CKAN permite a publicação de conjuntos de dados de formas simples, e conta com ferramentas robustas para o gerenciamento dos dados publicados e o monitoramento de sua utilização, bem como, uma interface que permite a visualização e extração dos dados publicamente, facilitando o acesso aos dados por qualquer um. O CKAN também conta com um poderosa API de fácil utilização, que pode ser utilizada para obter conjuntos de dados nos formatos mais utilizados em sistemas de informação tal como CSV, TSV, JSON ou XML e obtidos através de consultas estruturadas em JSON ou até mesmo SQL. Desta forma, a API pode ser aproveitada para que outros desenvolvedores utilizem a ferramenta em suas respectivas aplicações e desenvolvam soluções para fortalecer ainda mais a iniciativa de dados governamentais abertos.

\section{Revisão da Literatura}

\subsection{Desafios em relação à inovação baseada em dados}

Através de entrevistas semi-estruturadas com 7 empresas de setores de Tecnologia da Informação, Automobilística e de Telecomunicações sobre os desafios da inovação orientada à dados, o estudo de [Cronholm et al. 2017] identificou 5 categorias de desafios mais latentes ao contexto destas empresas. As categorias identificadas foram a falta de um processo sistemático, problemas com acesso a dados, desconfiança em relação a dados, falta de ferramentas adequadas e falta de competência.

A falta de processo sistemático refere-se ao fato de não existir um sistema estabelecido que conduza a inovação baseada em dados o que leva à falta de aprendizagem com os erros das iniciativas que ocorrem de forma singular e inconsistência na solidificação do processo. A categoria de problemas de acesso aos dados tem como principais causas

\footnotetext{
${ }^{2}$ https://ckan.org/
} 
a distribuição e fragmentação dos dados e a falta de gerenciamento destes, tendo como consequências a ineficiente coleta de dados e a obstrução na sua utilização que por sua vez geram problemas à tomada de decisão. A desconfiança em relação ao dados gera baixa confiança no resultado da análise dos dados e tem como principais consequências a complexa interpretação das representações visuais dos dados, a dificuldade no gerenciamento e definição dos objetivos chave e a dificuldade no estabelecimento dos fatores críticos de sucesso. A falta de competência é descrita através da falta de motivação das equipes e perda de competitividade gerada pela falta de treinamentos e as conclusões obtidas pelo problema de falta de ferramentas fortalecem o objetivo deste trabalho indicando a ineficiência na inovação e a lacuna por ferramentas de análise e visualização automáticas.

Estas categorias foram agrupadas numa categoria de contexto mais amplo que define que os dados são raramente utilizados como um recurso estratégico no processo de inovação orientada à dados. As consequências deste desafio são: (i) perda de informações do consumidor; (ii) reatividade, não proatividade; (iii) agravar as possibilidades de desenvolver soluções sustentáveis; (iv) qualidade inferior da base de decisão; (v) nenhum desenvolvimento da capacidade organizacional; (vi) possibilidades inexploradas de inovação de serviço; e (vii) fontes de dados valiosas permanecem subexploradas.

Uma conclusão importante desse estudo é falta de uma entidade responsável pelo armazenamento, acesso, gerenciamento, coleta e análise dos dados, o que impossibilita a exploração dos dados da organização. Essa conclusão é embasada por [Mathis 2015] que descreve o dilema das empresas no processo de inovação orientada por dados que é o fato de que os funcionários que possuem o conhecimento dos dados não estão engajados no processo de desenvolvimento estratégico de novos modelos de negócio e por outro lado, os gerentes que são os responsáveis pela parte de negócio não tem conhecimento dos dados disponíveis. Outra conclusão muito importante para fortalecer o objetivo desse trabalho é a falta de ferramentas digitais apropriadas, o que dificulta o uso de dados como um recurso estratégico. Esse desafio é descrito como um tema recorrente nas entrevistas e a necessidade de ferramentas é suportada por [Lee et al. 2014], que afirmam: "No atual ambiente de negócios competitivo, as empresas enfrentam desafios ao lidar com questões de big data, como a rápida tomada de decisões para melhorar a produtividade. Muitos sistemas de fabricação não estão prontos para gerenciar big data devido à falta de ferramentas analíticas inteligentes".

\subsection{Ferramenta de recomendação de visualização REV}

A ferramenta de visualização REV [Macedo et al. 2020] tem como proposta a automatização do processo de recomendação de visualização de dados abertos governamentais. $\mathrm{O}$ framework proposto pela ferramenta consiste basicamente em identificar os tipos de dados do conjunto de dados, transformá-los através de operações de compartimentação e agrupamento e em seguida gerar a visualização dos tipos de visualizações recomendadas [Barcellos et al. 2018]. Na primeira parte do processo o usuário deve inserir um conjunto de dados no formato ".csv". Em seguida a ferramenta identifica os tipos de dados de todas as colunas do dataset e os classifica em três categorias: dados categóricos, categóricos de data (temporais) ou quantitativos. Após essa etapa, os dados categóricos (temporais ou não) são dispostos para escolha do usuário no campo "Variável X" e os quantitativos dispostos nos campos "Variável X" e "Variável Y". Após a escolha das colunas por parte do usuário a ferramenta segue com o processo 
de transformação dos dados através das operações de compartimentação e agrupamento que são definidas por [Qin et al. 2018] como: (i) Compartimentação (binning): consiste no processo de separar o dado em categorias menores. Valores temporais são particionados em dia, mês e/ou ano. Valores numéricos são particionados com base em intervalos consecutivos, por exemplo: bin $1[0,10)$, bin2 [10,20] e (ii) Agrupamento: consiste em agrupar os valores quantitativos baseados nos valores categóricos correspondentes.

Após a etapa de transformação os dados de um mesmo grupo, ou seja, que possuem eixo categórico em comum (categoria, mês, data), podem passar por uma etapa de agregação onde são executados procedimentos como soma dos valores ou contagem do total de valores de uma coluna correspondente. Baseada na ferramenta TABLEAU Public $^{3}$, a ferramenta também propõe um tratamento diferenciado para eixos categóricos de data que visa possibilitar as operações de drill-down, que é a possibilidade de visualização em menor granularidade, e rollup que é a visualização dos dados dispostos em maior granularidade. A última etapa consiste na escolha das visualizações de acordo com os tipos de dados das colunas selecionadas pelo usuário. A ferramenta limita-se à recomendação de 4 tipos de visualizações, sendo eles:

- Gráficos de barras: recomendado quando o subconjunto de dados consiste em uma coluna categórica e outra quantitativa.

- Gráfico de pizza: recomendado quando o subconjunto de dados consistem em uma coluna categórica e outra quantitativa tendo a coluna categórica menos de 5 valores em sua totalidade.

- Gráfico de linha: recomendado quando o subconjunto de dados possui um eixo categórico temporal e um eixo quantitativo.

- Gráfico de dispersão: recomendado quando o subconjunto de dados possui os dois eixos compostos por colunas de tipo de dado quantitativo.

Ou seja, no trabalho [Macedo et al. 2020], os autores elucidam, de forma mais completa, os critérios adotados e apresentam os fundamentos utilizados na seleção do melhor tipo de visualização para cada tipo de dados. Utilizamos o framework apresentado em [Macedo et al. 2020] na construção da ferramenta móvel proposta neste trabalho.

\subsubsection{Open Data Companion}

Devido à facilidade de uso e praticidade trazida pelos dispositivos móveis o modo como as pessoas consomem e compartilham informações foi alterado. Os smartphones oferecem uma gama vasta de serviços e aplicações realmente úteis que, cada vez mais, se mostram parte essencial do desenvolvimento da sociedade. Contudo, ferramentas de dados abertos encontram-se em falta nas aplicações disponíveis. Tendo como principal objetivo aumentar a disponibilidade e acessibilidade de dados abertos na Internet a Utopia Software juntamente com a plataforma CKAN desenvolveu o Open Data Companion (ODC), um aplicativo mobile que reunia mais de 120 portais de dados abertos e milhares de datasets de diversas organizações espalhadas pelo mundo [Foundation 2015]. O aplicativo foi elaborado com design otimizado para dispositivos móveis e servia como uma maneira fácil e conveniente de encontrar, acessar e compartilhar dados abertos. O aplicativo

\footnotetext{
${ }^{3}$ https://public.tableau.com/pt-br/s/
} 
também fornecia a possibilidade de administradores de instâncias CKAN fornecer acesso aos seus dados sem custo ou trabalho árduo de configuração pois utilizava a poderosa api do CKAN que possibilitava integração automática com qualquer portal que rodasse a versão 2.0 ou mais atual a partir de uma extensão. Infelizmente o aplicativo parece ter sido descontinuado visto que até a data de confecção deste trabalho o link disposto na postagem do blog do CKAN encontra-se indisponível e o aplicativo não se encontra mais disponível na Play Store [Foundation 2015].

\section{Ferramenta Proposta}

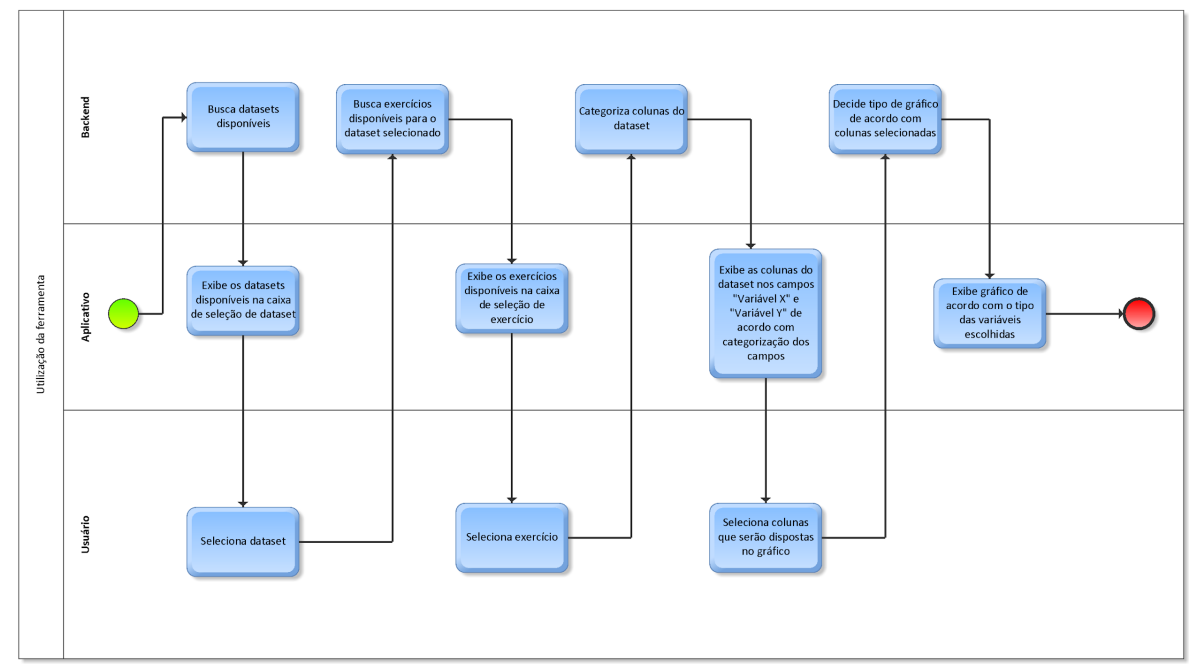

Figura 1. Processo da utilização da ferramenta em notação BPMN

Tendo em vista que o trabalho do gestor consiste em tomar a melhor decisão na imprecisão de informações que lhe estão disponíveis, ferramentas de suporte à tomada de decisão visam dirimir a ambivalência das informações e suas consequências negativas [Laudon et al. 2015]. De acordo com [Farazmand et al. 2018] sistemas de dados integrados que coletam informações podem ajudar os gerentes a obter percepções adicionais sobre os problemas, além de utilizar dados históricos para rastrear e revisar tendências, contextualizar os níveis de resultados atuais e definir prioridades com base na direção positiva ou negativa tomada pelos principais fatores de decisão. Tendo evidenciado a importância da gestão orientada a dados e o papel do gestor no processo de inovação orientada a dados, nos capítulos anteriores deste trabalho, construímos um aplicativo mobile para a visualização dados abertos, utilizando sistema de recomendação de visualização REV para a construção das visualizações. O nosso aplicativo visa incentivar a utilização de ferramentas de análise de dados, por parte dos gestores públicos e aproximá-los de dados que muitas vezes podem estar perdidos em arquivos extensos ou relatórios confusos.

O aplicativo móvel foi construído utilizando o framework Nativescript-Angular que utiliza um backend desenvolvido com o framework Quarkus (Java) para processar os dados dos datasets utilizados. O Nativescript é um framework de código aberto que permite a construção de aplicativos móveis para as plataformas Android e IOS de forma nativa e o Quarkus é um framework Java que reúne as melhores bibliotecas e padrões da plataforma Java. O código do aplicativo móvel está disponível neste repositório e do backend utilizado para processar os dados neste repositório . A ferramenta utiliza a API da 
instância CKAN do portal de dados abertos do município de Recife para extrair os dados dos datasets de Receitas, Despesas e Licitações em andamento e Licitações Concluídas dos exercícios 2018, 2019 e 2020. As tecnologias para o desenvolvimento da ferramenta foram escolhidas devido a familiaridade do autor na utilização da maioria das tecnologias citadas.

Para a construção das visualizações foi utilizada a biblioteca nativa do Nativescript que permite a construção de visualizações de diversos tipos. Por conta da natureza da visualização de dados em aparelhos móveis, a biblioteca oferece de forma nativa funções como pan e zoom e também atua fazendo a compartimentação(binning ) dos dados de forma automática. A biblioteca também permite a customização das características visuais das visualizações geradas tal como cor dos rótulos, tamanho das legendas e espessura de linhas. Embora demonstre-se bastante versátil e funcional, a biblioteca ainda apresenta algumas limitações para implementações mais avançadas e possui uma curva de aprendizado muito grande. Na Figura 1, apresentamos os passos da utilização da ferramenta de forma geral.

Inicialmente, ao abrir o aplicativo o sistema verifica os datasets disponíveis e os exibe na na caixa de seleção de dataset. A tela de seleção de datasets é apresentada na Figura 2(a). Após selecionar o dataset o sistema verifica os anos de exercício disponíveis para o dataset escolhido e os exibe na caixa de seleção de exercícios. A tela de seleção de exercícios é apresentada na Figura 2(b). Tendo definido o dataset e o exercício, o backend inicia o processo de categorização dos campos de acordo com o framework proposto pela ferramenta REV. Após serem devidamente categorizados, os campos quantitativos são dispostos na caixa de seleção "Variável X" e "Variável Y" e os categóricos são dispostos na caixa de seleção "Variável X". A tela de seleção dos campos "Variável X" e "Variável Y" são apresentadas, respectivamente, na Figura 3(a) e na Figura 3(b). Com os campos selecionados, o botão "Gerar gráfico" torna-se visível para que o usuário finalize a interação, exibido na Figura 4(a).

Nesse momento as informações selecionadas pelo usuário são enviadas para o backend da aplicação, onde este aplica o algoritmo da ferramenta REV para a escolha de qual tipo de visualização gerar. Após obter os dados e definir o tipo de visualização a ser gerada o aplicativo móvel verifica se existe eixo do tipo categórico, para realizar os métodos de agrupamento e soma dos dados. A compartimentação de eixos categóricos do tipo temporal é feita automaticamente pela biblioteca de visualizações do Nativescript, sem a necessidade de análise dos valores de data que estão sendo manipulados. Na Figura 4(b) apresentamos o gráfico gerado, onde o usuário consegue dar zoom, navegar pelo gráfico arrastando a tela horizontalmente ou verticalmente (panning) e alterar a orientação da tela do dispositivo para alterar o formato de visualização.

\section{Considerações Finais}

Neste trabalho propomos um aplicativo móvel que busca disponibilizar visualizações de qualidade baseadas no framework de recomendação da ferramenta REV, visando aprimorar a tomada de decisão baseada em dados e promover o acesso livre à dados governamentais abertos de forma prática. Uma solução mobile certamente possui limitações naturais por conta de seu tamanho, entretanto, por conta da evolução do comportamento da sociedade na execução de tarefas, aplicativos móveis podem tornar uma tarefa mais es- 


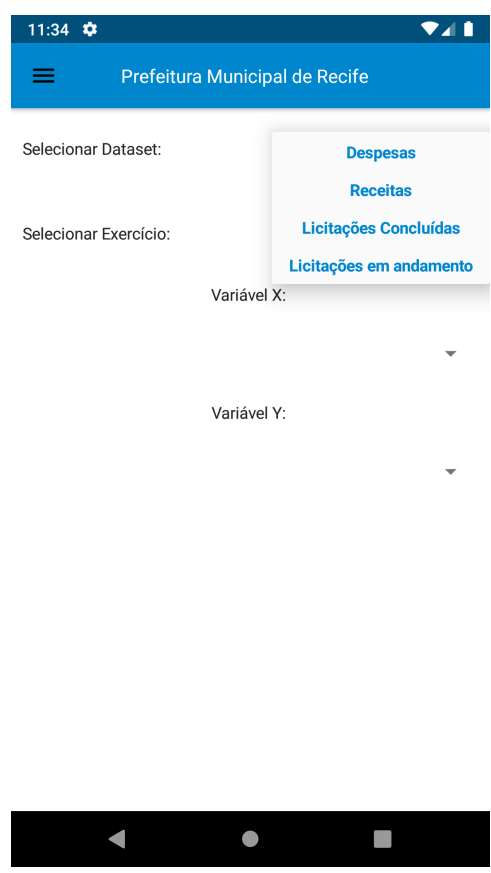

(a) Tela de seleção de dataset
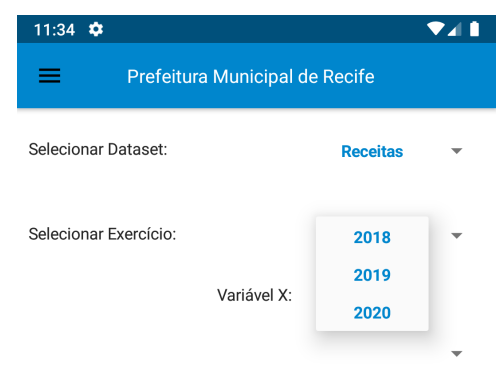

Variável Y:

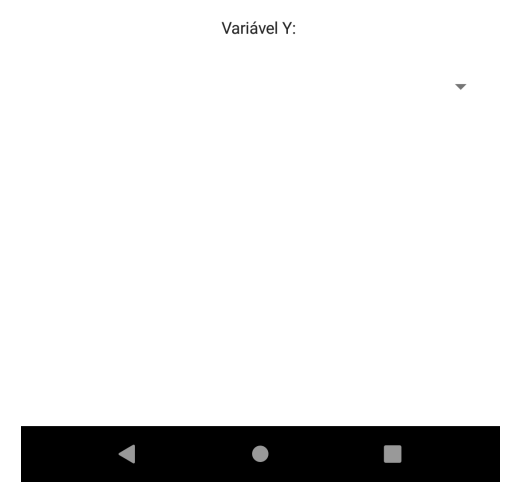

(b) Tela de seleção de exercício

\section{Figura 2. llustrações da ferramenta proposta, considerando seleção de dataset e de exercício}

timulante e engajante devido à sua usabilidade e ubiquidade. Dessa forma, a ferramenta proposta pode contribuir incentivando a tomada de decisão orientada a dados como uma ferramenta desktop, tal como a ferramenta REV não poderia, porque exige pouco esforço para entregar informações relevantes que possam trazer insights significativos para o gestor. Nesse mesmo contexto, a ferramenta também busca amenizar o problema de falta de ferramentas de análise de dados descrito anteriormente neste estudo. Outro ponto interessante da solução é a integração com o Portal de dados abertos do município de Recife que demonstra a possibilidade de adoção da ferramentas semelhantes por parte dos entes públicos e acaba por incentivar a adoção de uma padrão na publicação de dados abertos. O governo é a ferramenta pela qual melhoramos a vida dos cidadãos, portanto, este não deveria se atrasar ao implementar ações e ferramentas que podem melhorar a execução desta função. Isto posto, fica clara a necessidade de investimento em ferramentas com objetivo semelhante a desenvolvida neste trabalho.

A primeira versão da ferramenta proposta mostrou-se de fato coerente com os objetivos deste trabalho, entretanto, alguns pontos podem ser melhor desenvolvidos e lapidados a fim de tornar o trabalho mais relevante para a comunidade e obter melhores resultados na sua utilização. Este estudo delimita o gestor público como público-alvo da ferramenta proposta por conta de sua importância no contexto de inovação orientada a dados descrito anteriormente, contudo, ele poderia ser utilizado por cidadãos como fonte de informação sobre as ações do governo. Para tanto, seria interessante a execução de entrevistas e testes de usabilidade com usuários comuns para atestar sua utilização. A utilização do portal de dados abertos do município de Recife teve como prerrogativa a delimitação do trabalho para o contexto municipal, dado que este contexto possui maio- 


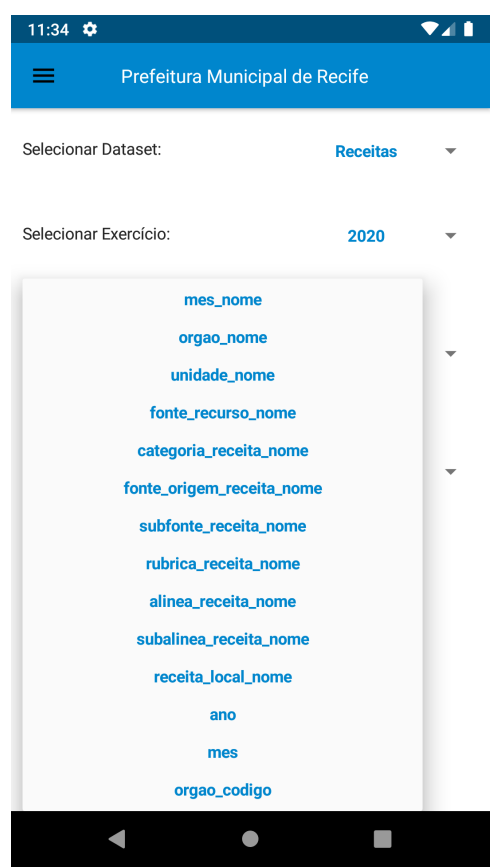

(a) Tela de seleção do campo "Variável X"
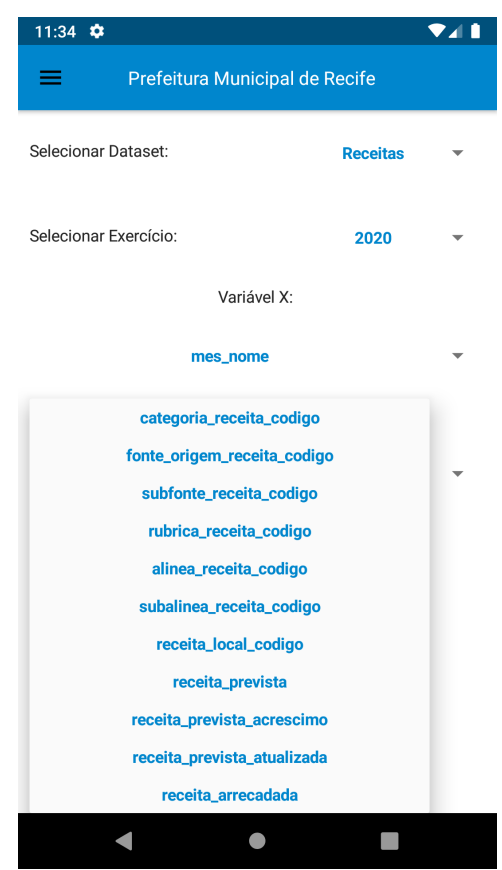

(b) Tela de seleção do campo "Variável Y"

Figura 3. llustrações da ferramenta proposta, considerando seleção do campo "Variável X" e "Variável Y"

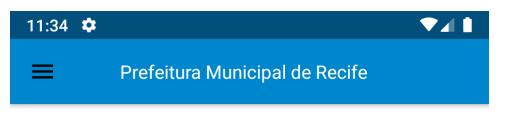

Selecionar Dataset:

Selecionar Exercício:

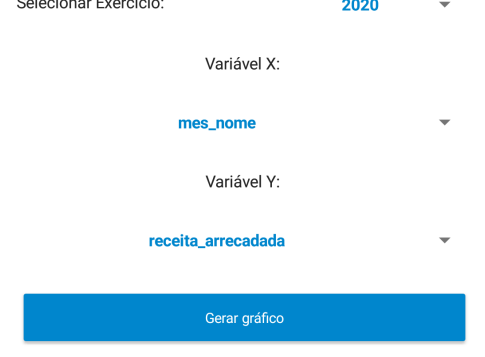

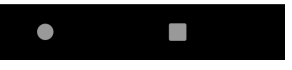

(a) Tela com o botão "Gerar gráfico" visível
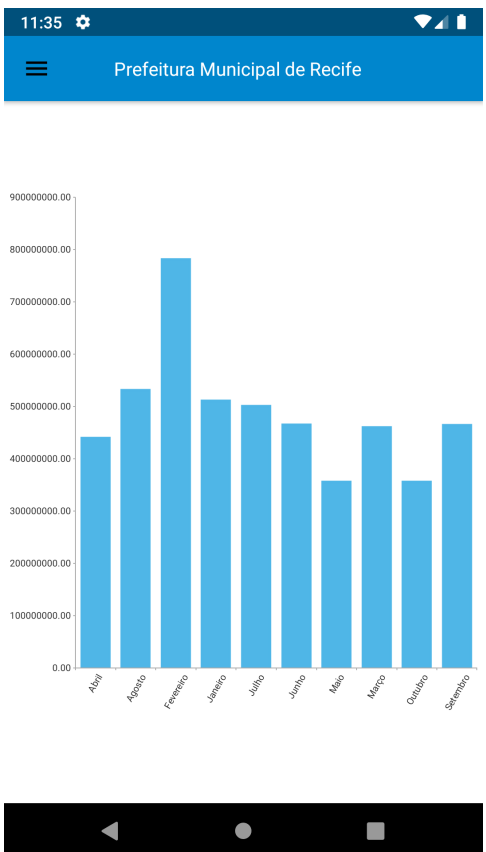

(b) Tela com o gráfico gerado

Figura 4. Ilustrações da ferramenta proposta, considerando tela com o botão "Gerar gráfico" visíve e tela com o gráfico geradol 
res peculiaridades. Contudo, a possibilidade de utilização de qualquer instância de portal de dados abertos que utilize o CKAN tornaria a solução mais abrangente. Além disso, a implementação de uma integração agnóstica permitiria a visualização dos dados de qualquer dataset disponível num determinado portal.

Como trabalho futuro, outro ponto a ser desenvolvido tem relação com a implementação do algoritmo de recomendação da ferramenta REV. A implementação atual permite apenas a utilização do algoritmo para os datasets previamente definidos. Para que este algoritmo seja utilizado em outros trabalhos, torna-se fundamental a disponibilização de uma API que tivesse como parâmetros uma fonte de dados de uma instância de CKAN e os campos a serem avaliados. Outro trabalho futuro interessante seria a realização de entrevistas com os gestores públicos a fim de dirimir a limitação deste estudo relacionada a falta de entrevistas com o público-alvo da solução proposta.

\section{Referências}

Andrews, L. (2019). Public administration, public leadership and the construction of public value in the age of the algorithm and 'big data'. Public Administration, 97(2):296310 .

Barcellos, R., Viterbo, J., Bernardini, F., and Trevisan, D. (2018). An instrument for evaluating the quality of data visualizations. In 2018 22nd International Conference Information Visualisation (IV), pages 169-174.

Bernardini, F., Viterbo, J., Cappelli, C., and Berger, M. (2020). As cidades do futuro e a computação. In Maciel, C. and Viterbo, J., editors, Computação e Sociedade - Volume 2 - A Sociedade, chapter 11, pages 81-107. EdUFMT, Cuiabá.

Bommert, B. (2010). Collaborative innovation in the public sector. Inter public management review, 11(1):15-33.

Brandão, S. M. and Bruno-Faria, M. d. F. (2017). Barreiras à inovação em gestão em organizações públicas do governo federal brasileiro: análise da percepção de dirigentes.

Cronholm, S., Göbel, H., and Rittgen, P. (2017). Challenges concerning data-driven innovation. In The 28th Australasian Conference on Information Systems, Hobart Australia, December 4-6, 2017.

Farazmand, A. et al. (2018). Global encyclopedia of public administration, public policy, and governance. Springer New York, NY.

Ferreira, B. (2019). Impulsionando inovação: novos designs para a gestão pública. Editora Bambual.

Foundation, O. K. (2015). Open data companion (odc) - bringing open data to the mobile platform. Available at https://okfnlabs.org/blog/2015/09/04/ bringing-open-data-to-mobile.html.

Guenduez, A. A., Mettler, T., and Schedler, K. (2020). Technological frames in public administration: What do public managers think of big data? Government Information Quarterly, 37(1):101406.

Gurstein, M. B. (2011). Open data: Empowering the empowered or effective data use for everyone? First Monday. 
Jetzek, T., Avital, M., and Bjorn-Andersen, N. (2014). Data-driven innovation through open government data. Journal of theoretical and applied electronic commerce research, 9(2):100-120.

Keim, D. A., Mansmann, F., Schneidewind, J., and Ziegler, H. (2006). Challenges in visual data analysis. In Tenth Inter Conf on Information Visualisation (IV'06), pages 9-16. IEEE.

Kerr do Amaral, H. and Licio, E. C. (2008). O desenvolvimento de dirigentes como estratégia para o fortalecimento da capacidade de governo no brasil: a experiência da enap. In XIII Congreso Internacional del CLAD.

Laudon, K. C., Laudon, J. P., et al. (2015). Management information systems. Pearson Upper Saddle River.

Lee, J., Kao, H.-A., and Yang, S. (2014). Service innovation and smart analytics for industry 4.0 and big data environment. Procedia Cirp, 16:3-8.

Macedo, D., Barcelos, R., Bernardini, F., and Viterbo, J. (2020). Uma ferramenta para recomendação de visualização de dados governamentais abertos. In Anais do VIII Workshop de Computação Aplicada em Governo Eletrônico, pages 96-107. SBC.

Machado, V., Mantini, G., Viterbo, J., Bernardini, F., and Barcellos, R. (2018). An instrument for evaluating open data portals: A case study in brazilian cities. In Proceedings of the 19th Annual Inter Conf on Digital Government Research: Governance in the Data Age, pages 1-10.

Magnusson, J., Koutsikouri, D., and Päivärinta, T. (2020). Efficiency creep and shadow innovation: enacting ambidextrous it governance in the public sector. European Journal of Information Systems, 29(4):329-349.

Mathis, K. (2015). Data-driven business models for service innovation in small and medium-sized businesses.

Mergel, I. (2018). Open innovation in the public sector: drivers and barriers for the adoption of challenge. gov. Public Management Review, 20(5):726-745.

Nicholson-Crotty, S., Nicholson-Crotty, J., and Fernandez, S. (2017). Performance and management in the public sector: Testing a model of relative risk aversion. Public Administration Review, 77(4):603-614.

Paraskevopoulos, P., Dinh, T.-C., Dashdorj, Z., Palpanas, T., and Serafini, L. (2013). Identification and characterization of human behavior patterns from mobile phone data. D4D Challenge session, NetMob.

Qin, X., Luo, Y., Tang, N., and Li, G. (2018). Deepeye: An automatic big data visualization framework. Big data mining and analytics, 1(1):75-82.

Zuiderwijk, A. and Janssen, M. (2014). Open data policies, their implementation and impact: A framework for comparison. Government Information Quarterly, 31(1):1729. 